\title{
HAM Gene Family and Shoot Meristem Development
}

\author{
Yuan Geng ${ }^{1,2}$ and Yun Zhou ${ }^{1,2 *}$ \\ 'Department of Botany and Plant Pathology, Purdue University, West Lafayette, IN, United States, ${ }^{2}$ Purdue Center for Plant \\ Biology, Purdue University, West Lafayette, IN, United States
}

Land plants develop highly diversified shoot architectures, all of which are derived from the pluripotent stem cells in shoot apical meristems (SAMs). As sustainable resources for continuous organ formation in the aboveground tissues, SAMs play an important role in determining plant yield and biomass production. In this review, we summarize recent advances in understanding one group of key regulators - the HAIRY MERISTEM (HAM) family GRAS domain proteins - in shoot meristems. We highlight the functions of HAM family members in dictating shoot stem cell initiation and proliferation, the signaling cascade that shapes HAM expression domains in shoot meristems, and the conservation and diversification of HAM family members in land plants. We also discuss future directions that potentially lead to a more comprehensive view of the HAM gene family and stem cell homeostasis in land plants.

OPEN ACCESS

Edited by:

Xigang Liu,

Hebei Normal University, China

Reviewed by:

Bo Sun,

Nanjing University, China Sascha Biedermann,

University of Freiburg, Germany

${ }^{*}$ Correspondence:

Yun Zhou

zhouyun@purdue.edu

Specialty section:

This article was submitted to Plant Physiology,

a section of the journal

Frontiers in Plant Science

Received: 22 October 2021 Accepted: 19 November 2021 Published: 20 December 2021

Citation:

Geng Y and Zhou Y (2021) HAM

Gene Family and Shoot

Meristem Development.

Front. Plant Sci. 12:800332.

doi: 10.3389/fpls.2021.800332
Keywords: shoot meristems, stem cells, land plants, HAM, GRAS proteins, microRNAs

\section{HAM KEEPS SHOOT STEM CELLS UNDIFFERENTIATED}

Land plants develop diversified shoot architectures, which are determined and sustained by pluripotent stem cells in shoot apical meristems (SAMs). In seed plants, the multicellular SAMs are organized into distinct cell layers and zones (Foster, 1938; Satina et al., 1940; Meyerowitz, 1997). In the model species Arabidopsis and many other flowering plants, SAMs consist of three clonally distinct cell layers: the epidermal layer (L1), the sub-epidermal layer (L2), and the corpus (L3) (Figure 1). In addition, SAMs can be divided into different functional zones, including the central zone $(\mathrm{CZ})$ where the self-renewing stem cells reside, the peripheral zone (PZ) where organ initiation takes place, and the rib meristem (RM) where the differentiated cells help specify the overlaying stem cells (Meyerowitz, 1997). Over more than 20 years of studies, multiple key regulatory pathways, such as the WUSCHEL-CLAVATA loop, KNOX/ SHOOTMERISTEMLESS pathway, ERECTA family receptors, Class III HD-ZIP transcription factors, and the cytokinin and auxin signaling, have been identified and well characterized in Arabidopsis SAMs (Sablowski, 2007; Barton, 2010; Su et al., 2011; Shpak, 2013; Gaillochet and Lohmann, 2015; Somssich et al., 2016; Fletcher, 2018; Kieber and Schaller, 2018; Shi and Vernoux, 2021; Willoughby and Nimchuk, 2021). In this review, we focus on the function and regulation of one group of conserved stem cell regulators, the HAIRY MERISTEM (HAM) family GRAS (GAI, RGA, and SCR) domain proteins, in shoot meristems.

The HAIRY MERISTEM (HAM) gene was firstly identified and characterized in Petunia, and it was named after the phenotype of its loss-of-function mutant, the ectopic formation of differentiated hairs (trichomes) at the surface of shoot apices (Stuurman et al., 2002). 

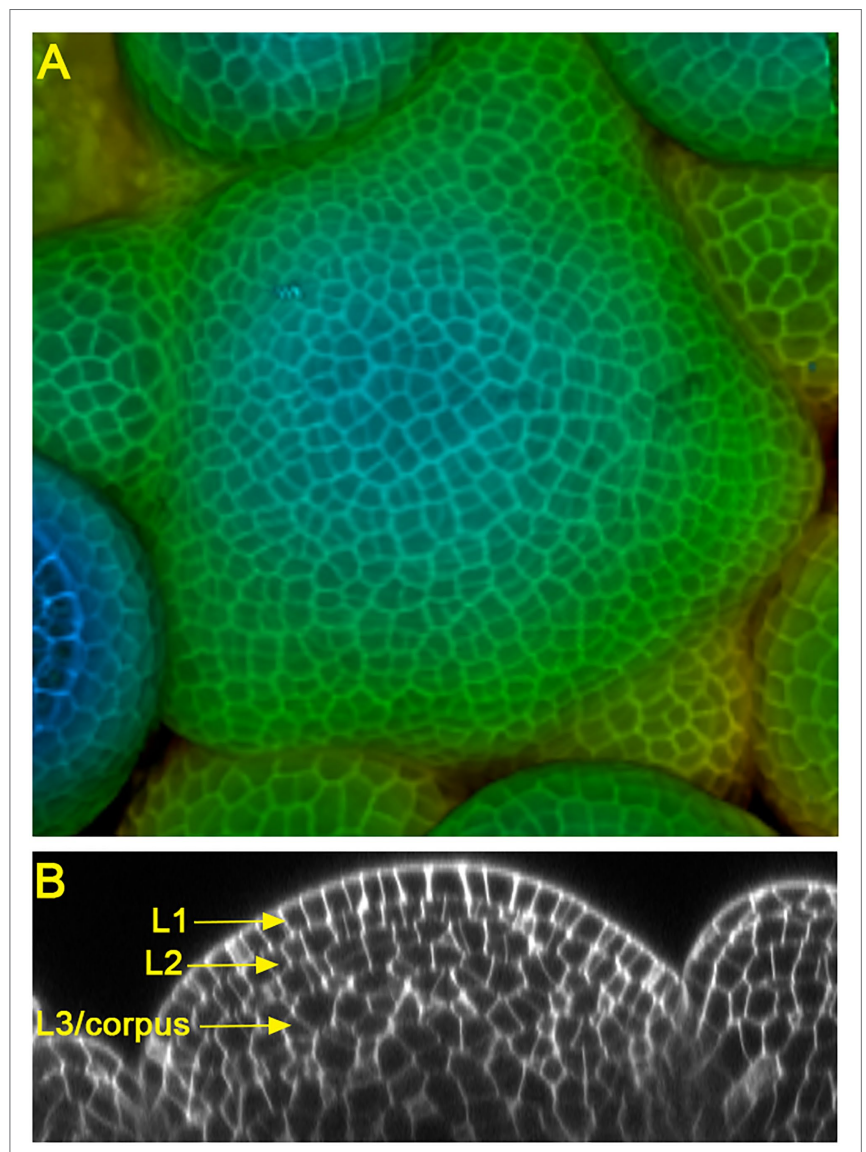

FIGURE 1 | Confocal images of Arabidopsis inflorescence shoot apical meristems (SAMs). (A) The 3D projection view of an Arabidopsis SAM, with the depth color coding. Blue represents the top surface layer and red represents the deepest layer. (B) The orthogonal view of an Arabidopsis SAM, showing three clonally distinct cell layers: the epidermal layer (L1), subepidermal layer (L2), and corpus (L3).

The HAM loss-of-function in Petunia also shows early termination of SAMs, arrested axillary shoot development, and reduced number of carpels and stamens (Stuurman et al., 2002), suggesting the key role of HAM in maintaining shoot meristems undifferentiated in Petunia. In the model species Arabidopsis, four HAM homologs (HAM1-HAM4) are classified into two different groups, based on the phylogenetic analyses (Engstrom et al., 2011; Geng et al., 2021b). HAM1, HAM2, and HAM3, which are also named as LOST MERISTEM1 (LOM1), LOM2, and LOM3, respectively (Schulze et al., 2010), belong to the Type II group (Engstrom et al., 2011; Geng et al., 2021b). These Type II members (HAM1-3) are expressed in Arabidopsis shoot meristems, root meristems, and vascular tissues (Schulze et al., 2010; Engstrom et al., 2011; Zhou et al., 2015). HAM4, the only member of the Type I group in Arabidopsis (Engstrom et al., 2011; Geng et al., 2021b), is specifically expressed in the provascular and vascular tissues (Zhou et al., 2015), sharing redundant function with HAM1-3 during shoot and root development (Engstrom et al., 2011; Zhou et al., 2015).

The Type II HAM members (HAM1, HAM2, and HAM3) play both overlapping and distinct roles in control of Arabidopsis
SAMs. The single loss-of-function mutant of each Type II member does not result in any obvious defects in Arabidopsis shoot meristem development (Schulze et al., 2010; Engstrom et al., 2011). By contrast, the ham 1ham2ham3 (ham123) triple loss-of-function mutant or the ham1ham2 (ham12) double mutant showed delayed inflorescence initiation, early termination of shoot meristems, disorganized meristem structure and morphology, and reduced axillary shoot branches (Schulze et al., 2010; Wang et al., 2010; Engstrom et al., 2011; Han et al., 2020a), demonstrating essential and redundant roles of Type II members in meristem initiation and maintenance in Arabidopsis. A recent study further shows that HAM1 and HAM2, both of which are expressed in the L3 layer, are required for maintaining SAMs undifferentiated and driving de novo formation of new axillary stem cell niches (Han et al., 2020a). HAM3, the other member of the Type II group, plays a minor role in shoot stem cell maintenance but likely contributes to other aspects of shoot development (Han et al., 2020a).

\section{HAM SUSTAINS THE WUSCHEL- CLAVATA REGULATORY LOOP}

In Arabidopsis, the homeobox domain transcription factor WUSCHEL (WUS) and the secreted peptide CLAVATA3 (CLV3) form a negative feedback loop to keep a constant population of stem cells in SAMs (Schoof et al., 2000; Somssich et al., 2016; Fletcher, 2018; Figure 2A). The WUS transcripts are restricted into the organizing center (OC) in deep cell layers (Mayer et al., 1998) and WUS proteins move into stem cells in the central zone to activate CLV3 expression (Schoof et al., 2000; Yadav et al., 2011; Daum et al., 2014). On the contrary, the CLV3 peptide, secreted from stem cells, activates the CLV receptor signaling pathways and confines WUS transcripts to the OC to avoid overproliferation of stem cells (Schoof et al., 2000). The ability of WUS to directly activate its own inhibitor CLV3 brings a potential risk to shut down itself and the feedback loop; therefore, the precise spatial-temporal regulations of WUS and $C L V 3$ are required for stem cell maintenance.

Several studies demonstrated that Type II HAM members play essential roles in initiating and maintaining the WUS-CLV3 feedback loop, and further sustaining shoot stem cell homeostasis in Arabidopsis (Schulze et al., 2010; Zhou et al., 2015, 2018; Gruel et al., 2018; Han et al., 2020a; Geng et al., 2021b), which also has been summarized in the reviews (Biedermann and Laux, 2018; Han et al., 2020b). Through the screening of an Arabidopsis transcription factor library, Type II HAM proteins are identified as the WUS interacting partners (Zhou et al., 2015). Among them, both HAM1 and HAM2 are co-expressed with WUS in the L3 layer. HAM1/2 act as WUS transcriptional cofactors to regulate the downstream targets and drive proliferation of shoot stem cells (Zhou et al., 2015). In addition, the expression patterns of HAM1/2 and CLV3 are largely complementary in Arabidopsis SAMs (Zhou et al., 2018). CLV3 is highly expressed in the L1 and L2 layers of the central zone, where HAM1 and HAM2 are absent or barely detectable (Zhou et al., 2018; Han et al., 2020a). These results lead to a 


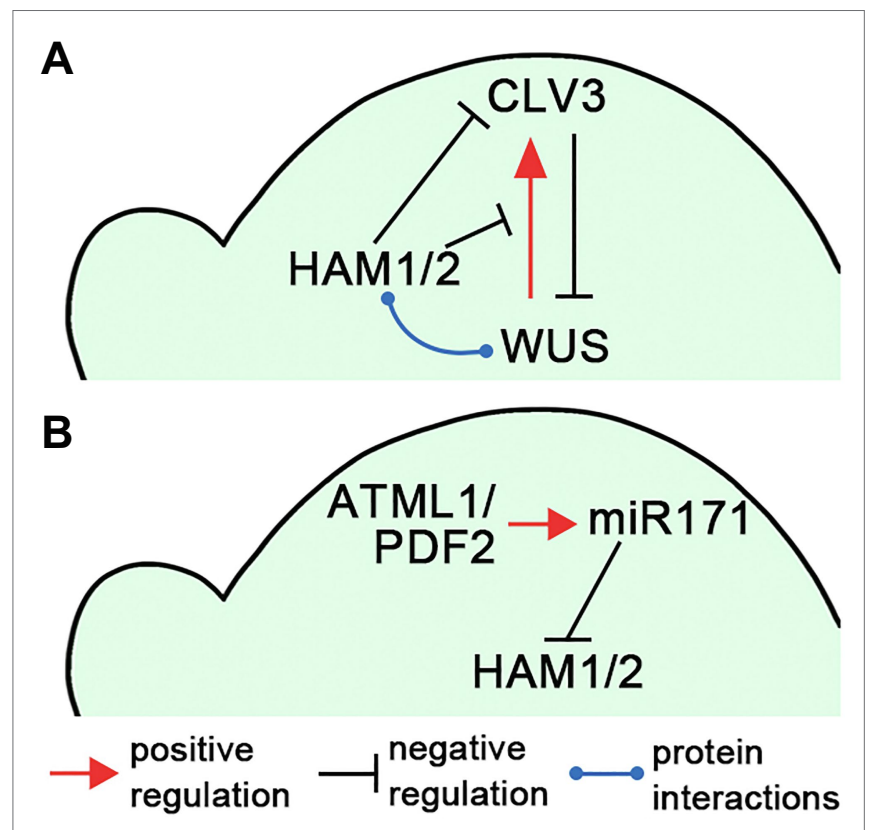

FIGURE 2 | Illustrations of the HAM regulatory circuits in SAMs.

(A) A diagram illustrates that HAM1/2 sustain the WUS-CLV3 feedback loop in Arabidopsis SAMs. (B) A diagram illustrates the L1(ATML1/PDF2)-miR171HAM signaling cascade, which shapes HAM1/2 expression patterns in Arabidopsis SAMs. The positive and negative regulations and protein-protein interactions are indicated in $\mathbf{( A , B )}$.

hypothesis that HAM1/2 together with WUS determine the $C L V 3$ expression pattern and confine the CLV3 domain to the stem cells in the outer layers of SAMs (Zhou et al., 2018; Han et al., 2020b; Figure 2A). Specifically, WUS protein activates CLV3 in the central zone where HAM1/2 proteins are absent, and HAM1/2 keep CLV3 off in the rib meristem, preventing WUS-dependent activation of CLV3 and/or repressing CLV3 transcription (Zhou et al, 2018). This working model has been supported by (Zhou et al., 2018; Han et al., 2020a; Geng et al., 2021b) and aligns with (Brand et al., 2000, 2002; Schoof et al., 2000; Graf et al., 2010; Schulze et al., 2010) a number of experimental results. It is also shown plausible by several independent computational simulations (Gruel et al., 2018; Zhou et al., 2018; Liu et al., 2020). In addition, through confocal imaging of HAM translational reporters and genetic complementation analyses, recent work shows that both HAM1 and HAM2 proteins, which show highly comparable expression patterns in the L3 layer of SAMs, are necessary and sufficient for determining the CLV3 pattern (Han et al., 2020a). HAM3, which is only expressed in the boundary between the meristem and primordia and at a few cells of the peripheral zone, is dispensable in shaping the CLV3 domain (Han et al., 2020a). In contrast, when HAM3 is expressed in the rib meristem under the control of the HAM2 promoter, it rescues the ectopic expression of CLV3 in the ham123 triple mutant (Han et al., 2020a), suggesting HAM3 protein maintains the function interchangeable with that of HAM1 and HAM2.

During the de novo formation of shoot stem cell niches, the expression patterns of HAM1/2 are dynamically regulated, which drive the switch of the CLV3 expression domain from the basal to apical region of developing axillary meristems over time (Zhou et al., 2018). In contrast, the expression of CLV3 is restricted to the basal part of developing axillary meristems in the ham 123 mutant, consistent with the mutant defects in axillary bud initiation (Schulze et al., 2010; Wang et al., 2010; Engstrom et al., 2011; Zhou et al., 2018).

\section{A SIGNALING CASCADE SHAPES HAM PATTERNS IN ARABIDOPSIS SHOOT MERISTEMS}

In Arabidopsis, a small group of micro RNAs - the microRNA171 (miR171) - function as the negative regulator of Type II HAM members (Llave et al., 2002; Rhoades et al., 2002; Schulze et al., 2010; Wang et al., 2010; Engstrom et al., 2011; Han et al., 2020c). miR171 specifically recognizes and binds to Arabidopsis $H A M 1, H A M 2$, and HAM3, mediating the cleavage of their transcripts (Llave et al., 2002; Rhoades et al., 2002). Consistently, MIR171 overexpression leads to ectopic expression of CLV3 in the rib meristem and reduced shoot branching, which mimic the phenotype of the ham123 mutant (Schulze et al., 2010; Wang et al., 2010; Zhou et al., 2018; Han et al., 2020a).

The epidermis-derived miR171 defines the apical-basal concentration gradient of HAM1/2 in Arabidopsis SAMs and axillary meristems (Takanashi et al., 2018; Han et al., 2020c). Four MIR171 family genes (MIR171A, MIR171B, MIR171C, and $M I R 170$ ) are identified in Arabidopsis, all producing miR171 precursors and contributing to the total level of mature miR171 (Llave et al., 2002; Rhoades et al., 2002). All these MIR171/170 genes are directly activated by the homeodomain transcription factor ARABIDOPSIS THALIANA MERISTEM LAYER 1 (ATML1) and its close homolog PROTODERMAL FACTOR 2 (PDF2) in the L1 layer (Han et al., 2020c). Once synthesized in the epidermis, mature miR171 moves downwards within limited distance and it mediates the cleavage of the transcripts of HAM1-3 in the apical region of SAMs (Han et al., 2020c). Based on these results, a L1(ATML1/PDF2)-miR171-HAM signaling cascade has been proposed, which initiates and then maintains the apical-basal concentration gradient of Type II HAM proteins in Arabidopsis shoot meristems (Han et al., 2020c; Figure 2B). The essential function of the L1-miR171-HAM signaling cascade is simulated by a computational model and further validated by in vivo experimentations including the time-lapse live imaging upon the transient activation of ATML1 in the SAMs (Han et al., 2020c).

\section{EVOLUTION OF HAM GENE FAMILY IN LAND PLANTS}

The phylogenetic analysis suggested that the HAM gene family emerged during the divergence of land plant lineages (Geng et al., 2021b). In non-flowering plants including bryophytes, lycophytes, ferns, and gymnosperms, HAM members are maintained with a low copy number (Engstrom et al., 2011; Geng et al., 2021b). 
By contrast, the HAM gene family likely duplicated in a common ancestor of flowering plants, expanding to two diversified groups (Type I and Type II) as mentioned above, in flowering plants (Geng et al., 2021b). Type II HAM members are widely present in flowering plants, whereas Type I HAM members were independently lost in the species from different orders (including Poales and Asparagales) in monocots (Geng et al., 2021b).

HAM family members from several flowering plants share similar functions in maintaining indeterminacy of SAMs and promoting de novo formation of axillary meristems (Stuurman et al., 2002; Schulze et al., 2010; Wang et al., 2010; Engstrom et al., 2011; David-Schwartz et al., 2013; Zhou et al., 2015, 2018; Hendelman et al., 2016). For example, the ham loss-of-function mutant in pepper (Capsicum annuum) shows the shoot meristem defect (David-Schwartz et al., 2013) comparable to that characterized in the Petunia ham mutant and in the Arabidopsis ham123 mutant (Stuurman et al., 2002; Schulze et al., 2010; Engstrom et al., 2011). Several HAM homologs, including AmHAM1 (the Type I) and AmHAM2 (the Type II) from Amborella trichopoda (the species as a sister group to all other flowering plants), one Type II HAM from a monocot (rice), and two Type II HAM members from eudicots (soybean and pepper), are able to replace the role of Arabidopsis Type II HAM members in Arabidopsis shoot meristems (Geng et al., 2021b), demonstrating the conserved function of HAM family members in flowering plants.

The results from cross-species complementation assays also indicate the conserved biochemical function between the non-flowering HAM proteins and the Type II HAM proteins from flowering plants, in regulating meristem development (Geng et al., 2021b). When different non-flowering HAM members (including PpHAM from the bryophyte Physcomitrium (Physcomitrella) patens, SmHAM from the lycophyte Selaginella moellendorffii, CrHAM from the fern Ceratopteris richardii, and LkHAM from the gymnosperm Larix kaempferi) are expressed under the control of the Arabidopsis HAM2 promoter, they replace the function of Type II members (HAM1, HAM2, and HAM3) in regulating the CLV3 expression domain, maintaining established SAMs, and promoting the initiation of new stem cell niches in Arabidopsis ham 123 mutants (Geng et al., 2021b). Consistently, the function of PpGRAS12/PPHAM was also characterized in the moss Physcomitrium (Physcomitrella) patens (Beheshti et al., 2021). Overexpression of PpGRAS12 leads to formation of supernumerary apical meristems on each gametophore, suggesting a positive role of PpGRAS12/PpHAM in control of stem cell population at the gametophyte stage (Beheshti et al., 2021). Taken together, all the current results lead to a hypothesis that regulation of stem cell homeostasis is an ancestral and conserved trait of the HAM gene family, which deserves more functional studies of HAM homologs in land plants, especially in seedfree plants. Recent advances in the genomic and transcriptomic resources (Marchant et al., 2019; Geng et al., 2021a), established transformation system (Plackett et al., 2014) and quantitative confocal imaging platform (Wu et al., 2021) in seed-free vascular plants, such as in Ceratopteris richardii, will facilitate us to test this hypothesis and further understand meristem evolution in land plants.

\section{CONSERVATION AND DIVERSIFICATION OF THE MIR171-HAM REGULATION IN LAND PLANTS}

The phylogenetic analysis and sequence alignment demonstrate that the 21-nt miR171 binding site (5'-GATATTGGCGCGGC TCAATCA-3') is highly conserved within the coding sequences of the non-flowering HAM members and the majority of Type II HAM members in flowering plants (Engstrom et al., 2011; Geng et al., 2021b). The negative regulation of Type II HAM members by miR171 seems to be conserved in flowering plants as well. For example, transcripts of two HAM family genes (SlHAM1 and SlHAM2) in tomato (Solanum lycopersicum) and four HAM homologs in rice (Oryza sativa) are also specifically targeted and cleaved by miR171 (Fan et al., 2015; Hendelman et al., 2016). Overexpression of MIR171 genes in tomato and rice results in reduced expression of these HAM homologs and the disruption of meristem development (Fan et al., 2015; Hendelman et al., 2016).

Furthermore, when the non-flowering HAM members (such as PpHAM, SmHAM, CrHAM, and LkHAM) and several Type II HAM members from flowering plants (including Amborella, the monocot rice, and the dicot soybean and pepper) are expressed under the control of Arabidopsis HAM2 promoter, these HAM reporters showed the concentration gradient from low to high along the apical-basal axis of Arabidopsis SAMs (Geng and Zhou, 2021; Geng et al., 2021b). These expression patterns are largely comparable to that of the miR171-sensitive HAM2 translational reporter (Han et al., 2020a; Geng et al., 2021b); however, they are different from that of the miR171-insenstive HAM2 transcriptional reporter, which shows high expression in all the cells from different layers in Arabidopsis SAMs (Han et al., 2020a). These findings suggest a conserved role of the miR171 binding sites in the non-flowering HAM members and in the majority of Type II HAM members from flowering plants.

Different from the Type II, Type I HAM genes show different extents of diversification in the miR171 binding site (Engstrom et al., 2011; Geng et al., 2021b). Based on the sequence alignment (Geng et al., 2021b), only a few Type I HAM members (such as AmHAM1 from Amborella trichopoda and the HAM homologs from Nelumbo nucifera and Vitis vinifera) maintain the conserved miR171 binding site, and many others from a considerable number of flowering plants lost the conservation of the miR171 binding site. For example, HAM4 (the Arabidopsis Type I HAM) contains six nucleotides different from the conserved miR171 binding sequence and is unlikely targeted by miR171 (Engstrom et al., 2011; Geng et al., 2021b).

\section{FUTURE PERSPECTIVES}

Over the last several years, significant progress has been made in understanding the functions of Type II HAM members in shoot meristems and their interaction with the WUS-CLV3 loop, the regulatory mechanism by which Type II HAM proteins are excluded from stem cells in Arabidopsis SAMs, and evolution of different groups of HAM members in land plants. In the 
future, several important questions are still remaining to be explored. For example, in Arabidopsis SAMs, in contrast to WUS and CLV3 that are specifically expressed in a few cells, HAM1 and HAM2 proteins are expressed in a broader domain (Zhou et al., 2015, 2018; Han et al., 2020a). It will be interesting to explore whether the Type II HAM members also integrate additional and multiple regulatory pathways in control of shoot stem cells. In addition, the L1-miR171-HAM signaling cascade plays a crucial role during the initiation and maintenance of Arabidopsis shoot meristems (Han et al., 2020c). It will be worth determining whether this signaling cascade also functions in other meristematic tissues in Arabidopsis and whether this regulatory mechanism is conserved across flowering plants or even in non-flowering plants. Furthermore, the function of Type I HAM members is not completely understood yet. Determining whether and how this group of HAM members have been recruited into various developmental processes and undergone neofunctionalization in land plants will be an essential question in the future.

\section{REFERENCES}

Barton, M. K. (2010). Twenty years on: the inner workings of the shoot apical meristem, a developmental dynamo. Dev. Biol. 341, 95-113. doi: 10.1016/j. ydbio.2009.11.029

Beheshti, H., Strotbek, C., Arif, M.A., Klingl, A., Top, O., and Frank, W. (2021). PpGRAS12 acts as a positive regulator of meristem formation in Physcomitrium patens. Plant Mol. Biol. doi: 10.1007/s11103-021-01125-z, [Epub ahead of print].

Biedermann, S., and Laux, T. (2018). Plant development: adding HAM to stem cell control. Curr. Biol. 28, R1261-R1263. doi: 10.1016/j.cub.2018.09.039

Brand, U., Fletcher, J. C., Hobe, M., Meyerowitz, E. M., and Simon, R. (2000). Dependence of stem cell fate in Arabidopsis on a feedback loop regulated by CLV3 activity. Science 289, 617-619. doi: 10.1126/science.289.5479.617

Brand, U., Grünewald, M., Hobe, M., and Simon, R. (2002). Regulation of CLV3 expression by two homeobox genes in Arabidopsis. Plant Physiol. 129, 565-575. doi: 10.1104/pp.001867

Daum, G., Medzihradszky, A., Suzaki, T., and Lohmann, J. U. (2014). A mechanistic framework for noncell autonomous stem cell induction in Arabidopsis. Proc. Natl. Acad. Sci. U. S. A. 111, 14619-14624. doi: 10.1073/ pnas. 1406446111

David-Schwartz, R., Borovsky, Y., Zemach, H., and Paran, I. (2013). CaHAM is autoregulated and regulates CaSTM expression and is required for shoot apical meristem organization in pepper. Plant Sci. 203-204, 8-16. doi: 10.1016/j.plantsci.2012.12.011

Engstrom, E. M., Andersen, C. M., Gumulak-Smith, J., Hu, J., Orlova, E., Sozzani, R., et al. (2011). Arabidopsis homologs of the Petunia HAIRY MERISTEM gene are required for maintenance of shoot and root indeterminacy. Plant Physiol. 155, 735-750. doi: 10.1104/pp.110.168757

Fan, T., Li, X., Yang, W., Xia, K., Ouyang, J., and Zhang, M. (2015). Rice Osa-miR171c mediates phase change from vegetative to reproductive development and shoot apical meristem maintenance by repressing four OsHAM transcription factors. PLoS One 10:e0125833. doi: 10.1371/journal. pone. 0125833

Fletcher, J. C. (2018). The CLV-WUS stem cell signaling pathway: a roadmap to crop yield optimization. Plants 7:87. doi: 10.3390/plants7040087

Foster, A. S. (1938). Structure and growth of the shoot apex in Ginkgo Biloba. Bull. Torrey. Bot. Club 65, 531-556. doi: 10.2307/2480793

Gaillochet, C., and Lohmann, J. U. (2015). The never-ending story: from pluripotency to plant developmental plasticity. Development 142, 2237-2249. doi: 10.1242/dev.117614

Geng, Y., Cai, C., Mcadam, S. A. M., Banks, J. A., Wisecaver, J. H., and Zhou, Y. (2021a). A de novo transcriptome assembly of Ceratopteris richardii provides insights into the evolutionary dynamics of complex gene families in land plants. Genome Biol. Evol. 13:evab042. doi: 10.1093/gbe/evab042

\section{AUTHOR CONTRIBUTIONS}

All authors listed have made a substantial, direct and intellectual contribution to the work, and approved it for publication.

\section{FUNDING}

This work was supported by the Purdue start-up package and National Science Foundation IOS-1931114 (to YZ), and the Purdue Center for Plant Biology Graduate Student Fellowship and Ross-Lynn Graduate Research Scholarship (to YG).

\section{ACKNOWLEDGMENTS}

We apologize to the colleagues whose work was not cited in this review due to the space limitation.

Geng, Y., Guo, L., Han, H., Liu, X., Ann Banks, J., Wisecaver, J. H., et al. (2021b). Conservation and diversification of HAIRY MERISTEM gene family in land plants. Plant J. 106, 366-378. doi: 10.1111/tpj.15169

Geng, Y., and Zhou, Y. (2021). N-terminal region is required for functions of the HAM family member. Plant Signal. Behav. 16:1940001. doi: 10.1080/15592324.2021.1940001

Graf, P., Dolzblasz, A., Würschum, T., Lenhard, M., Pfreundt, U., and Laux, T. (2010). MGOUN1 encodes an Arabidopsis type IB DNA topoisomerase required in stem cell regulation and to maintain developmentally regulated gene silencing. Plant Cell 22, 716-728. doi: 10.1105/tpc.109.068296

Gruel, J., Deichmann, J., Landrein, B., Hitchcock, T., and Jönsson, H. (2018). The interaction of transcription factors controls the spatial layout of plant aerial stem cell niches. NPJ Syst. Biol. Appl. 4:36. doi: 10.1038/s41540-018-0072-1

Han, H., Geng, Y., Guo, L., Yan, A., Meyerowitz, E. M., Liu, X., et al. (2020a). The overlapping and distinct roles of HAM family genes in Arabidopsis shoot meristems. Front. Plant Sci. 11:541968. doi: 10.3389/fpls.2020.541968

Han, H., Liu, X., and Zhou, Y. (2020b). Transcriptional circuits in control of shoot stem cell homeostasis. Curr. Opin. Plant Biol. 53, 50-56. doi: 10.1016/j. pbi.2019.10.004

Han, H., Yan, A., Li, L., Zhu, Y., Feng, B., Liu, X., et al. (2020c). A signal cascade originated from epidermis defines apical-basal patterning of Arabidopsis shoot apical meristems. Nat. Commun. 11:1214. doi: 10.1038/s41467-020-14989-4

Hendelman, A., Kravchik, M., Stav, R., Frank, W., and Arazi, T. (2016). Tomato HAIRY MERISTEM genes are involved in meristem maintenance and compound leaf morphogenesis. J. Exp. Bot. 67, 6187-6200. doi: 10.1093/jxb/erw388

Kieber, J. J., and Schaller, G. E. (2018). Cytokinin signaling in plant development. Development 145:dev149344. doi: 10.1242/dev.149344

Liu, Z., Shpak, E. D., and Hong, T. (2020). A mathematical model for understanding synergistic regulations and paradoxical feedbacks in the shoot apical meristem. Comput. Struct. Biotechnol. J. 18, 3877-3889. doi: 10.1016/j.csbj.2020.11.017

Llave, C., Xie, Z., Kasschau, K. D., and Carrington, J. C. (2002). Cleavage of scarecrow-like mRNA targets directed by a class of Arabidopsis miRNA. Science 297, 2053-2056. doi: 10.1126/science.1076311

Marchant, D. B., Sessa, E. B., Wolf, P. G., Heo, K., Barbazuk, W. B., Soltis, P. S., et al. (2019). The C-Fern (Ceratopteris richardii) genome: insights into plant genome evolution with the first partial homosporous fern genome assembly. Sci. Rep. 9:18181. doi: 10.1038/s41598-019-53968-8

Mayer, K. F. X., Schoof, H., Haecker, A., Lenhard, M., Jürgens, G., and Laux, T. (1998). Role of WUSCHEL in regulating stem cell fate in the Arabidopsis shoot meristem. Cell 95, 805-815. doi: 10.1016/S0092-8674(00)81703-1

Meyerowitz, E. M. (1997). Genetic control of cell division patterns in developing plants. Cell 88, 299-308. doi: 10.1016/S0092-8674(00)81868-1

Plackett, A. R. G., Huang, L., Sanders, H. L., and Langdale, J. A. (2014). High-efficiency stable transformation of the model fern species Ceratopteris 
richardii via microparticle bombardment. Plant Physiol. 165, 3-14. doi: 10.1104/pp.113.231357

Rhoades, M. W., Reinhart, B. J., Lim, L. P., Burge, C. B., Bartel, B., and Bartel, D. P. (2002). Prediction of plant microRNA targets. Cell 110, 513-520. doi: $10.1016 /$ S0092-8674(02)00863-2

Sablowski, R. (2007). The dynamic plant stem cell niches. Curr. Opin. Plant Biol. 10, 639-644. doi: 10.1016/j.pbi.2007.07.001

Satina, S., Blakeslee, A. F., and Avery, A. G. (1940). Demonstration of the three germ layers in the shoot apex of Datura by means of induced polyploidy in periclinal chimeras. Am. J. Bot. 27, 895-905. doi: 10.1002/j.1537-2197.1940. tb13952.x

Schoof, H., Lenhard, M., Haecker, A., Mayer, K. F. X., Jürgens, G., and Laux, T. (2000). The stem cell population of Arabidopsis shoot meristems is maintained by a regulatory loop between the CLAVATA and WUSCHEL genes. Cell 100, 635-644. doi: 10.1016/S0092-8674(00)80700-X

Schulze, S., Schäfer, B. N., Parizotto, E. A., Voinnet, O., and Theres, K. (2010). LOST MERISTEMS genes regulate cell differentiation of central zone descendants in Arabidopsis shoot meristems. Plant J. 64, 668-678. doi: 10.1111/j.1365-313X.2010.04359.x

Shi, B., and Vernoux, T. (2021). Hormonal control of cell identity and growth in the shoot apical meristem. Curr. Opin. Plant Biol. 65:102111. doi: 10.1016/j. pbi.2021.102111

Shpak, E. D. (2013). Diverse roles of ERECTA family genes in plant development. J. Integr. Plant Biol. 55, 1238-1250. doi: 10.1111/jipb.12108

Somssich, M., Je, B. I., Simon, R., and Jackson, D. (2016). CLAVATA-WUSCHEL signaling in the shoot meristem. Development 143, 3238-3248. doi: 10.1242/ dev. 133645

Stuurman, J., Jäggi, F., and Kuhlemeier, C. (2002). Shoot meristem maintenance is controlled by a GRAS-gene mediated signal from differentiating cells. Genes Dev. 16, 2213-2218. doi: 10.1101/gad.230702

Su, Y.-H., Liu, Y.-B., and Zhang, X.-S. (2011). Auxin-cytokinin interaction regulates meristem development. Mol. Plant 4, 616-625. doi: 10.1093/mp/ ssr007

Takanashi, H., Sumiyoshi, H., Mogi, M., Hayashi, Y., Ohnishi, T., and Tsutsumi, N. (2018). miRNAs control HAM1 functions at the single-cell-layer level and are essential for normal embryogenesis in Arabidopsis. Plant Mol. Biol. 96, 627-640. doi: 10.1007/s11103-018-0719-8
Wang, L., Mai, Y. X., Zhang, Y. C., Luo, Q., and Yang, H. Q. (2010). MicroRNA171ctargeted SCL6-II, SCL6-III, and SCL6-IV genes regulate shoot branching in Arabidopsis. Mol. Plant 3, 794-806. doi: 10.1093/mp/ssq042

Willoughby, A. C., and Nimchuk, Z. L. (2021). WOX going on: CLE peptides in plant development. Curr. Opin. Plant Biol. 63:102056. doi: 10.1016/j. pbi.2021.102056

Wu, X., Yan, A., Mcadam, S. A. M., Banks, J. A., Zhang, S., and Zhou, Y. (2021). Timing of meristem initiation and maintenance determines the morphology of fern gametophytes. J. Exp. Bot. 72, 6990-7001. doi: 10.1093/ jxb/erab307

Yadav, R. K., Perales, M., Gruel, J., Girke, T., Jonsson, H., and Reddy, G. V. (2011). WUSCHEL protein movement mediates stem cell homeostasis in the Arabidopsis shoot apex. Genes Dev. 25, 2025-2030. doi: 10.1101/ gad.17258511

Zhou, Y., Liu, X., Engstrom, E. M., Nimchuk, Z. L., Pruneda-Paz, J. L., Tarr, P. T., et al. (2015). Control of plant stem cell function by conserved interacting transcriptional regulators. Nature 517, 377-380. doi: 10.1038/nature13853

Zhou, Y., Yan, A., Han, H., Li, T., Geng, Y., Liu, X., et al. (2018). HAIRY MERISTEM with WUSCHEL confines CLAVATA3 expression to the outer apical meristem layers. Science 361, 502-506. doi: 10.1126/science.aar8638

Conflict of Interest: The authors declare that the research was conducted in the absence of any commercial or financial relationships that could be construed as a potential conflict of interest.

Publisher's Note: All claims expressed in this article are solely those of the authors and do not necessarily represent those of their affiliated organizations, or those of the publisher, the editors and the reviewers. Any product that may be evaluated in this article, or claim that may be made by its manufacturer, is not guaranteed or endorsed by the publisher.

Copyright $\odot 2021$ Geng and Zhou. This is an open-access article distributed under the terms of the Creative Commons Attribution License (CC BY). The use, distribution or reproduction in other forums is permitted, provided the original author(s) and the copyright owner(s) are credited and that the original publication in this journal is cited, in accordance with accepted academic practice. No use, distribution or reproduction is permitted which does not comply with these terms. 